\title{
An optimization framework for classifier learning from image data for computer-assisted diagnosis
}

\author{
J. Mennicke ${ }^{1}$, C. Münzenmayer ${ }^{2}$, T. Wittenberg ${ }^{2}$, and U. Schmid \\ ${ }^{1}$ Faculty Information Systems and Applied Computer Science, University of Bamberg, Bamberg, Germany \\ ${ }^{2}$ Dept. for Image Processing \& Biomedical Engineering, Fraunhofer-Institute for Integrated Circuits IIS, Erlangen, Germany
}

\begin{abstract}
In computer-assisted medical diagnosis it is often hard or even impossible to obtain a valid set of rules for disease classification by classical knowledge engineering methods. Alternatively, machine learning methods are applied to obtain classifiers from sets of data pre-classified by medical experts. Typically in a medical context, available data sets are imbalanced with respect to the possible classifications. E.g., in dermatology, there are only few data representing cases of malign melanoma vs. many cases representing benign nevi. Furthermore, there are different missclassification costs assigned to different classes. E.g., it is much more critical (i.e. costly) to erroneously classify a malign melanoma as benign than the other way around. We propose a universally applicable optimization framework that successfully corrects the error-based inductive bias of classifier learning methods on image data. The framework integrates several techniques of common optimization techniques, such as modifying the optimization procedure for inducer-specific parameters, modifying input data by an arcing algorithm, combining classifiers of several classifier learning methods ( $\mathrm{kNN}, \mathrm{SVM}$ and $\mathrm{C4.5})$ with different settings according to locally-adaptive, cost-sensitive voting schemes. The framework is designed to make the learning process cost-sensitive and enforcing more balanced missclassification costs between classes. The framework was evaluated on image data for Barrett's esophagus with promising results compared to the base learners.
\end{abstract}

Keywords-Classifier Learning, Computer-Assisted Diagnosis, Medical Image Data.

\section{INTRODUCTION}

When designing a system for computer-assisted diagnosis - be it in medicine or in other contexts of application - the greatest challenge is to model the expert knowledge in such a way that automated diagnosis (a) meets the accuracy of human experts and (b) the criteria on which diagnosis was based are transparent to the human expert. The traditional way to design a diagnosis system is knowledge engineering, that is, to employ empirical methods of knowledge acquisition and to build the knowledge base by formalizing this knowledge. This approach is known to be tedious and prone to errors and omissions - the so called knowledge acquisition bottleneck [Cohen and Feigenbaum, 1982], [Cuilen and Bryman, 1988]. An alternative way to obtain expert knowledge is to use machine learning methods [Mitchell, 1997]. Here, experts give their diagnoses for a sample of medical data. The data are represented as feature vectors and the diagnoses are coded as classes and associated with the according feature vector. The great advantage of the learning approach to knowledge acquisition is that the experts are just doing what they are experts in - that is, come up with a diagnosis in light of medical data - and there is no need to conduct lengthy interviews or other assessment methods. A classification learner will automatically extract the relevant feature combinations which best reproduce the diagnostic ratings of the experts and the learned classifier subsequently can be used to generate diagnostic proposals.

In many areas of medicine, the main data sources on which diagnoses are based are visual: In the most simple case data result from direct inspection of skin, throat, teeth or other body parts. Often image data are gained from X-rays or camera snapshots (e.g. in endoscopy). Obtaining textural, color or form features from such image data is a much researched topic in the domain of computer-assisted medical diagnosis [Münzenmayer, 2006]. Typically, the main effort in designing and implementing such diagnosis systems is to come up with fast and reliable algorithms for feature extraction from image data. In contrast, modeling of the classification rules which are applied to such features to produce a diagnosis is often done in a rather ad hoc manner. This aspect is the focus of the work presented in this paper and, as argued above, we propose using machine learning methods to obtain classification rules by which a diagnosis can be gained from features extracted from image data.

A common problem of machine learning approaches is that the quality of the obtained classifiers is dependent on a fairly even distribution of cases between classes. In the context of medical diagnosis, typically severe illnesses occur very seldomly and therefore, the data sample is biased. Furthermore, in medical diagnostics faulty diagnoses vary in their degree of harmfulness for the patients: Overseeing a severe illness is highly critical while erroneously suspecting a severe illness which can be exluded after further examinations is relatively harmless. Finally, diagnostic classes often 
have varying degrees of hardness of class boundaries, that is, only for some types of data, diagnosis is straight-forward.

To address these problems, we designed a framework considering several points for improvement of common optimization techniques, such as modifying the optimization procedure for inducer-specific parameters, modifying input data implemented by an arcing algorithm, and combining classifiers of several classifier learning methods with different settings according to locally-adaptive, cost-sensitive voting schemes. The framework is designed to make the learning process cost-sensitive and enforcing more balanced misclassification costs between classes.

In the following we first introduce the medical image data used to evaluate our framework. Afterwards we shortly characterize the base machine learning methods used. Afterwards we present our learning framework, followed by the learning results gained by using this framework versus the base learners. Details about the data, further data sets we investigated, the background of classifier learning, the framework, and the experimental settings and results are given in [Mennicke et al., 2008].

\section{Data Collections}

As data base we used two sets of endoscopic images from patients with suspected Barrett's mucosa [Münzenmayer, 2006]. The Barrett's esophagus classification problem (data sets I233P1017 and I183P1035) requires the framework to induce a classifier that successfully classifies mucous tissues into one of three different classes: Class $B E$ represents Benign Epithelium, class $B M$ Neoplastic Barrett's Mucosa and class $C C$ contains mucosa of the Cardia and Corpus as there is no clear boundary between these two.

For feature extraction from the images, color texture algorithms [Münzenmayer, 2006] were used. After feature extraction, each image is represented by a real-valued feature vector. The two data sets are different in the features extracted from these images. Features for data set I233P1017 were extracted using spectral color correction, color shading correction and statistical geometrical features [Münzenmayer, 2006]. Features for data set I183P1035 were extracted using color shading correction and sum- and difference-histograms [Münzenmayer, 2006]. The characteristics of the data sets are given in table 1 .

For the experiments, exemplary cost matrices were defined to our best knowledge (see table 2). For real applications, these matrices should be defined by experts.

The classes in these data sets are imbalanced. Furthermore, class boundaries have varying degrees of hardness
Table 1 Characteristics of data sets

\begin{tabular}{|l|l||l|l|}
\hline \multicolumn{2}{|c||}{ I183P1035 } & \multicolumn{2}{c|}{ I233P1017 } \\
\hline \hline \multicolumn{2}{|c|}{ 482 Cases } & \multicolumn{2}{c|}{ 300 Cases } \\
\hline \hline \multicolumn{2}{|c||}{ 180 Attributes } & \multicolumn{2}{c|}{ 48 Attributes } \\
\hline \hline Class & \# Cases & Class & \# Cases \\
\hline EP & 182 & EP & 97 \\
\hline BM & 122 & BM & 80 \\
\hline CC & 178 & CC & 123 \\
\hline
\end{tabular}

Table 2 Cost matrix of Barrett's esophagus classification problems

\begin{tabular}{|c||l|l|l|}
\hline \multicolumn{1}{|c||}{ True } & \multicolumn{3}{c|}{ Classified as (h(x)) } \\
Class c(x) & EP & BM & CC \\
\hline \hline EP & 0 & 0.25 & 0.25 \\
\hline BM & 0.75 & 0 & 1 \\
\hline CC & 0.25 & 0.5 & 0 \\
\hline
\end{tabular}

that correlate with the cost matrix, meaning that hard to learn classification boundaries (high misclassifications in the misclassification matrix) are also most costly. This poses classification problems which are difficult to handle by any error-based learning method.

\section{ClassifiER LEARNERS}

There exists a large variety of methods for classification learning with different strengthes and weaknesses [Michie et al., 1994]. We selected three well known learning approaches which are highly different. The high variety in the characteristics of the methods is expected to contribute to an overall improvement of the classification performance when combined into a single voting classifier. Taking local capabilities of the learners into account, such a meta learner should be capable of overcoming individual weaknesses while combining only the best aspects of the individual classifiers.

$K$-nearest neighbor learning ( $k N N)$ [Cover and Hart, 1967] is the most basic lazy (or instancebased) learning method [Mitchell, 1997]. Lazy learners, as opposed to eager learners, do not explicitly induce a representation of the target function. Their training phase rather consists of simply storing the training data. Generalization is postponed until an unseen query instance is to be classified. During the classification phase the learning algorithm examines the relationship of the new instance to the instances in training data. This forms the basis for the decision about the assigned class. $k N N$ learning can be expected to be less capable of defining class regions in feature space which suffer from the curse of dimensionality, meaning that a lot of attributes are irrelevant 
for defining the local decision boundary. Nevertheless, for class regions in feature space whose decision boundaries depend on many attributes, boundaries are possibly very complex and drawn diagonal to the attribute axes. $k N N$ can be expected to outperform other methods, because the classification is based on local instances only rather than on a global approximation of the target function. When highly complex decision boundaries need to be approximated, the value of $k$ must be small, thereby increasing the sensitivity to noise in data. The degree of fit to the data can only be adjusted globally.

Support vector machines (SVMs) [Schölkopf et al., 1999] are powerful kernel-based learning methods that belong to the class of eager learners. SVMs aim to induce a linear decision function in feature space. In a multi-dimensional feature space, such a linear decision function is represented by a hyperplane. The hyperplane is to be chosen in such a way that its margin is maximal. The margin is defined by the minimal distance of the hyperplane to those instances in training data (support vectors) which are closest to the hyperplane. As opposed to $k N N$ learning, $S V M$ s can be expected to suffer less from the curse of dimensionality and to class regions in which instances of neighboring classes are imbalanced. They are able to draw arbitrary decision functions in input space which yield a global approximation of the target function.

Decision Tree (DT) inducers are eager learning methods which build symbolic hypotheses represented by a decision tree (or a set of if-then-rules). With this type of hypothesis language disjunctive concepts can be expressed and the learned trees or rules are easily understandable to humans. One of the most common decision tree learning algorithms is C4.5 [Quinlan, 1993] which can be applied to problems with discrete as well as continuous-valued attributes. DT learning can be expected to suffer least from the curse of dimensionality, as it is the only method that considers the local discriminative power of the attributes. It is robust to noise in data, as most relevant attribute tests are based on many training instances, thereby reducing the effect of noise on the classification performance. The sensitivity of DTs to noise, when classifying an unseen instance on continuousvalued attributes, is eliminated by the application of soft thresholds in Quinlan's C4.5. The degree of fit to the data is adjusted locally by the pruning facilities according to the relevance of the attribute tests of each subtree. However, for complex decision boundaries that are drawn diagonal to the attribute axes, $D T$ s will only perform well if sufficient data is available. Additionally, DT learning is usually sensitive to imbalanced data sets, because the selection of attribute tests will be biased by such distributions in data.

\section{OPTIMIZATION FRAMEWORK}

To realize cost-sensitive learning and enforce more balanced misclassification costs between classes, we propose a framework which is organized in three stages:

- The base learning methods (here $k N N, S V N$, and $D T$ ) tuned by parameter selection with regard to error-based, cost-sensitive, and cost-balancing objectives (Level 1).

- A cost-sensitive, cost-balancing arcing (boosting-like) algorithm wrapped around each base learner that creates ensembles modifying inputs of the base learner in a cost-sensitive, cost-balancing manner and combines the models of the ensemble by a cost-sensitive, locally adaptive voting scheme. Several strategies are used for both input-modifications and voting (Level 2).

- A combination of such boosted ensembles again using cost-sensitive, locally-adaptive voting schemes (Level 3).

Cost-sensitive means that costs from a misclassification cost matrix are to be minimized. Cost-balancing refers to the capability of the classification to deliver balanced average expected misclassification costs between classes. Locallyadaptive can refer to the region in feature space, but in the experiments this only refers to being adaptive to the respective classes.

Arcing (adaptively resampling and combining) [Domingos, 1999] algorithms are an approach to optimize learners by introducing resampling techniques, training different classifiers with different samples and produce a classification via majority vote over these classifiers. For our framework we developed a new algorithm which allows for cost-sensitive and cost-balancing arcing and uses a locally adaptive combination scheme [Mennicke et al., 2008]. Resampling is realized via probabilities $p(i)$ associated with training data, making it more probable for a data vector with a high probability vector to be included into the sample.

Cost-sensitive resampling is based on the following weight updating formula:

$$
p_{t+1}(i)=\frac{p_{t}(i) *\left(1+\operatorname{cst}\left(c\left(x_{i}\right), h_{t}\left(x_{i}\right)\right)^{a}\right)}{\sum_{i}\left(p_{t}(i) *\left(1+\operatorname{cst}\left(c\left(x_{i}\right), h_{t}\left(x_{i}\right)\right)^{a}\right)\right)}
$$

Cost-sensitive and cost-balancing resampling is based on:

$p_{t+1}(i)=\frac{p_{t}(i) *\left(1+\operatorname{cst}\left(c\left(x_{i}\right), h_{t}\left(x_{i}\right)\right)^{a}\right) *\left(1+\left(\operatorname{cst}_{c\left(x_{i}\right)}\right)^{b}\right)}{\sum_{i} p_{t}(i) *\left(1+\operatorname{cst}\left(c\left(x_{i}\right), h_{t}\left(x_{i}\right)\right)^{a}\right) *\left(1+\left(\operatorname{cst}_{c\left(x_{i}\right)}\right)^{b}\right)}$

where

$$
\operatorname{cst}_{c\left(x_{i}\right)}=\frac{\sum_{x: c\left(x_{i}\right)==c(x)} \operatorname{cst}\left(c\left(x_{i}\right), h(x)\right)}{\sum_{x: c\left(x_{i}\right)==c(x)} 1}
$$


Table 3 Results of error-based $C 4.5, S V M$ and $k N N$ compared to average meta-learner

\begin{tabular}{|c|c|c|c|}
\hline Classifier & Error-based $C 4.5$ & Average Meta & Diff $(\%)$ \\
\hline Overall Error & 0.245 & 0.136 & 44.5 \\
\hline Deviation Costs & 0.182 & 0.130 & 28.6 \\
\hline Squared Sum Costs & 0.162 & 0.074 & 54.3 \\
\hline Overall Costs & 0.159 & 0.100 & 37.1 \\
\hline Classifier & Error-based $S V M$ & Average Meta & Diff (\%) \\
\hline Overall Error & $\overline{0.196}$ & 0.136 & 30.6 \\
\hline Deviation Costs & 0.151 & 0.130 & 13.9 \\
\hline Squared Sum Costs & 0.097 & 0.074 & 23.7 \\
\hline Overall Costs & 0.130 & 0.100 & 23.1 \\
\hline
\end{tabular}

\begin{tabular}{|l||l|l|l|}
\hline Classifier & Error-based $k N N$ & Average Meta & Diff (\%) \\
\hline \hline Overall Error & 0.174 & 0.136 & 21.8 \\
\hline Deviation Costs & 0.166 & 0.130 & 21.7 \\
\hline Squared Sum Costs & 0.116 & 0.074 & 36.2 \\
\hline Overall Costs & 0.123 & 0.100 & 18.7 \\
\hline
\end{tabular}

and $\operatorname{cst}\left(c\left(x_{i}\right), h_{t}\left(x_{i}\right)\right)$ represents the misclassification cost as predefined (see table 2).

We defined different voting schemes similar to a weighted product rule [Mennicke et al., 2008]. The most simple scheme is based on the actual costs caused by a classifier on all available data - that is, the classifier with the highest global trust is selected. To realize local adaptiveness, another voting scheme selects that classifier which performes best on a specific output class. By combining both schemes, a mixed trust scheme can be obtained which was also used.

Combining different strategies for parameter selection for the three base learners (level 1) with different strategies for resampling and voting (level 2) results in a collection of classifiers which are finally combined into a meta classifier (level 3). For the meta classifier the same voting schemes as on level 2 were realized.

\section{RESUlTS AND CONCLUSION}

The specific settings for the base algorithms as well for details on the sampling method and evaluation measures used are reported in [Mennicke et al., 2008]. Improvements achieved by the whole framework compared to the errorbased learning base methods $D T, S V M$, and $k N N$ on data sets I233P1017 and I183P1035 in all performance measures ranged from $13.9-54.3 \%$ (averaged over both data sets). The results are summarized in table 3 . In addition to the overall classification error, performance evaluation was done with respect to the costs associated with misclassifications.

In addition to the data reported here, we also performed experiments with image date for malign melanoma classification and blood cell classification with similar results.
Since medical image data have several characteristics which make it hard for standard error-based machine learning approaches to produce automated classifiers with acceptable accuracy rates such methods are often ignored and computer-assisted diagnosis systems rely on hand-crafted classification rules instead. We proposed a learning framework which specifically addresses the specifics of medical image data and could show promising initial results.

\section{REFERENCES}

Cohen and Feigenbaum, 1982. Cohen, P. R. and Feigenbaum, E. A. (1982). The Handbook of Artificial Intelligence, volume 3. William Kaufmann, Los Altos, CA.

Cover and Hart, 1967. Cover, T. and Hart, P. (1967). Nearest Neighbor Pattern Classification. IEEE Transactions on Information Theory, 13:21-27.

Cuilen and Bryman, 1988. Cuilen, J. and Bryman, A. (1988). The knowledge acquisition bottleneck: Time for a reassessment? ExPert Systems, 5(3):216-224.

Domingos, $1999 . \quad$ Domingos, P. (1999). MetaCost: a general method for making classifiers cost-sensitive. In KDD '99: Proceedings of the Fifth ACM SIGKDD International Conference on Knowledge Discovery and Data Mining, pages 155-164. ACM Press.

Mennicke et al., 2008. Mennicke, J., Münzenmayer, C., and Schmid, U. (2008). Classifier Learning for Imbalanced Data with Varying Misclassification Costs - A Comparison of kNN, SVM, and Decision Tree Learning. VDM, Saarbrücken. (based on the diploma thesis of J. Mennicke, University of Bamberg, 2006, http://www.cogsys.wiai.unibamberg.de/theses/mennicke/mennicke.pdf)

Michie et al., 1994. Michie, D., Spiegelhalter, D., and Taylor, C. (1994). Machine Learning, Neural and Statistical Classification. Ellis Horwood.

Mitchell, 1997. Mitchell, T. M. (1997). Machine Learning. McGrawHill, New York.

Münzenmayer, 2006. Münzenmayer, C. (2006). Color texture analysis in medical applications. Der Andere Verlag, Tönning.

Quinlan, 1993. Quinlan, J. R. (1993). C4.5: Programs for Machine Learning. Morgan Kaufmann, San Francisco.

Schölkopf et al., 1999. Schölkopf, B., Burges, C. J. C., and Smola, A. J. (1999). Advances in Kernel Methods - Support Vector Learning. MIT Press, Cambridge, MA.

\author{
Address of the corresponding author: \\ Author: Ute Schmid \\ Institute: Faculty WIAI, University of Bamberg \\ Street: Feldkirchenstraße 21 \\ City: 96045 Bamberg \\ Country: Germany \\ Email: ute.schmid@uni-bamberg.de
}

